\title{
Trace element compositions of fine- grained CAIs and AOAs in unaltered carbonaceous chondrites: Implications for early solar system reservoirs
}

\author{
SARA S RUSSELL ${ }^{1}$ AND ALEXANDER N. KROT ${ }^{2}$
}

${ }^{1}$ Natural History Museum, Cromwell Road, London SW7

5BD, UK, sarr@nhm.ac.uk

${ }^{2}$ HIGP, University of Hawai'i at Mānoa, Honolulu HI, 96822, USA.sasha@hawaii.edu

Fine-grained $\mathrm{Ca}$,Al-rich inclusions (CAIs) and amoeboid olivine aggregates (AOAs) are unmelted aggregates of solar nebula condensates. Fine-grained CAIs from Efremovka (CV3) are composed of spinel \pm hibonite \pm perovskite nodules each surrounded by melilite \pm hibonite \pm perovskite and/or anorthite and Al-diopside. The CAI cores and mantles are enriched in anorthite and melilite, respectively, suggesting multistage formation history in the nebular gas (Krot et al., 2004). Oxygen-isotope analyses show the CAIs were originally ${ }^{16} \mathrm{O}$-rich, but have experienced exhange with an ${ }^{16} \mathrm{O}$-depleted gaseous reservoir either in the nebula or on the CV chondrite parent body (Aléon et al., 2004).

We have analysed the rare earth element (REE) abundances of the Efremovka CAIs and AOAs by LA-ICPMS. The CAIs are all enriched in REEs, $\sim 10 \times \mathrm{CI}$, and have a fractionated Group II REE pattern (Boynton, 1975), indicating formation from a gaseous reservoir depleted in the most refractory (ultrarefractory) REEs. The REE patterns appear to have been not modified during subsequent metasomatic alteration affected $\mathrm{CV}$ chondrites. AOAs are characterized by flat (unfractionated) Group I REE patterns indicating that fine-grained CAIs and AOAs formed in chemically different nebular regions. One of the Efremovka fine-grained CAIs, E68, has a Group II REE pattern core surrounded by a Group I REE pattern mantle. The coexistence of the Group I and II REE patterns within a single CAI may indicate evolution of trace element abundances in the gaseous reservoirs recorded by this inclusion. The early solar system comprised compositionally separate reservoirs, evidence for which has also been recently reported in oxygen isotopes [4].

References: [1] Krot A. N. et al. (2004) Meteorit. Planet. Sci. 39, 1517-1553 [2] Aléon J. et al. (2005) Meteorit. Planet. Sci. 40, 1043-1058 [3] Boynton W. V. (1975) Geochim. Cosmochim. Acta 39, 569-584 [4] Bodénan J.-D. et al. (2020) Geochim. Cosmochim. Acta, in review. 